(NASA-CR-196805) IMPROVED IMAGE

DECOMPRESSION FOR REDUCED TRANSFORM CODING ARTIFACTS (Notre Dame Univ.) $10 \mathrm{p}$
N95-11761

Unclas

$63 / 610022363$ 


\title{
Improved image decompression for reduced transform coding artifacts
}

\author{
Thomas P. O'Rourke, Student Member, IEEE, and \\ Robert L. Stevenson, Member, IEEE
}

\begin{abstract}
The perceived quality of images reconstructed from low bit rate compression is severely degraded by the appearance of transform coding artifacts. This paper proposes a method for producing higher quality reconstructed images based on a stochastic model for the image data. Quantization (scalar or vector) partitions the transform coefficient space and maps all points in a partition cell to a representative reconstruction point, usually taken as the centroid of the cell. The proposed image estimation technique selects the reconstruction point within the quantization partition cell which results in a reconstructed image which best fits a non-Gaussian Markov random field (MRF) image model. This approach results in a convex constrained optimization problem which can be solved iteratively. At each iteration, the gradient projection method is used to update the estimate based on the image model. In the transform domain, the resulting coefficient reconstruction points are projected to the particular quantization partition cells defined by the compressed image. Experimental results will be shown for images compressed using scalar quantization of block DCT and using vector quantization of subband wavelet transform. The proposed image decompression provides a reconstructed image with reduced visibility of transform coding artifacts and superior perceived quality.
\end{abstract}

\section{INTRODUCTION}

Source coding of image data has been a very active area of research for many years. The goal is to reduce the number of bits needed to represent an image while making as few as possible perceptible changes to the image. Many algorithms have been developed which can successfully compress a grayscale image to around 0.8 bits per pixel (bpp) with almost no perceptible effects [1]. A problem arises, however, as these compression techniques are pushed beyond this rate. For higher compression ratios $(<0.4 \mathrm{bpp}$ for grayscale) most algorithms start to generate artifacts which severely degrade the perceived quality of the image. The type of artifacts generated is dependent on the compression technique and on the particular image. For block encoded images, the most noticeable artifact is generally the discontinuities present at the block boundaries. For subband encoded images, it is the ringing at image edges which is usually first perceived.

This paper proposes a technique which addresses this issue through a postprocessing algorithm which greatly re-

This paper has been submitted to IEEE Transactions on Circuits and Systems for Video Technology. This work was supported in by part by NASA Lewis Research Center under contract NASA-NAG 3-1540. This work was presented in part at the 1994 SPIE/IS\& T's Symposium on Electronic Imaging Science \& Technology, San Jose, CA, February, 1994

The authors are with the Laboratory for Image and Signal Analysis in the Department of Electrical Engineering at the University of Notre Dame, Notre Dame, IN 46555 . duces the artifacts introduced by the coding technique. It is based on a stochastic framework in which probabilistic models are used for both the noise introduced by the coding and for a "good" image. The restored image is the maximum a posteriori (MAP) estimate based on these models $[2 ; 3]$. Previous techniques which have tried to address this issue have various problems which limit their ability to produce high quality image estimates. Some techniques propose changes in the way the image is coded [4;5]; this however reduces the efficiency of the source coder and thus reduces the compression ratio. The proposed postprocessing technique only requires modification of the image decoder. Linear based estimators [6], while removing some artifacts, usually degrade edge information in the original image. Several techniques try to overcome this smoothing of the edges by first estimating the edge information in the compressed image data $[7 ; 8]$. This however is a very difficult task for very high compression ratios, where the actual edge information is somewhat scrambled.

This paper will first describe a generic model for image compression. For the purpose of the reconstruction algorithm, this model is descriptive enough to describe many compression techniques. A decompression algorithm is then described based on a previously proposed image model $[9 ; 10]$. The computational algorithm is also described. Experimental results are shown for two different transform coding methods. The first method is the Joint Photographic Experts Group (JPEG) image compression standard $[11 ; 1]$. The second method uses a subband wavelet transform with a vector quantization (VQ) proposed in [12]. It can be seen that images reconstructed with this new postprocessing technique show a reduction in many of the most noticeable artifacts and thus allow higher compression ratios.

\section{DECOMPRESSION ALGORITHM}

To decompress the compressed image representation, a MAP technique is proposed. Let the compressed image data be represented by $\mathbf{y}$ while the decompressed full resolution image is represented by $z$. For MAP estimation, the decompressed image estimate $z$ is given by

$$
\dot{z}=\arg \max _{\mathbf{z}} L(\mathbf{z} \mid \mathbf{y})
$$

where $L(\cdot)$ is the $\log$ likelihood function $L(\cdot)=\log \operatorname{Pr}(\cdot)$ which in this case is a measure of how likely the decompressed image $\mathbf{z}$ resulted in the compressed representation 
y. Using Bayes rule

$$
\begin{aligned}
\hat{\mathbf{z}} & =\arg \max _{\mathbf{z}}\left\{\log \frac{\operatorname{Pr}(\mathbf{y} \mid \mathbf{z}) \operatorname{Pr}(\mathbf{z})}{\operatorname{Pr}(\mathbf{y})}\right\}, \\
& =\arg \max _{\mathbf{z}}\{\log \operatorname{Pr}(\mathbf{y} \mid \mathbf{z})+\log \operatorname{Pr}(\mathbf{z})-\log \operatorname{Pr}(\mathbf{y})\}(3) \\
& =\arg \max _{\mathbf{z}}\{\log \operatorname{Pr}(\mathbf{y} \mid \mathbf{z})+\log \operatorname{Pr}(\mathbf{z})\},
\end{aligned}
$$

where the $\operatorname{Pr}(\mathbf{y})$ term is dropped because it is a constant with respect to the optimization parameter $\mathbf{z}$. The conditional probability $\operatorname{Pr}(\mathbf{y} \mid \mathbf{z})$ is based on the image compression method while the probability $\operatorname{Pr}(\mathbf{z})$ is based on prior information about the image data.

\section{A. Image compression model}

In a transform coding compression technique, a unitary transformation $H$ is applied to the original image $\mathbf{x}$. The compressed representation $\mathbf{y}$ is obtained by applying a quantization $Q$ to the transform coefficients which can be written as

$$
\mathbf{y}=Q[H \mathbf{x}]
$$

Quantization partitions the transform coefficient space and maps all points in a partition cell to a representative reconstruction point, usually taken as the centroid of the cell. The indices of these cells are usually entropy coded and then transmitted as the compressed representation $\mathbf{y}$. In the standard image decompression method, the reconstructed image is given by

$$
\hat{\mathbf{z}}=H^{-1} Q^{-1}[\mathbf{y}]
$$

where the inverse quantization maps the indices to the reconstruction points.

Since quantization is a many-to-one operation, many images map into the same compressed representation. The operation of the quantizer is assumed to be noise free; that is, a given image $\mathbf{z}$ will be compressed to the same compressed representation y every time. The conditional probability for the noise free quantizer can be described by

$$
\operatorname{Pr}(\mathbf{y} \mid \mathbf{z})= \begin{cases}1, & \mathbf{y}=Q[H \mathbf{z}] \\ 0, & \mathbf{y} \neq Q[H \mathbf{z}]\end{cases}
$$

Since

$$
\log \operatorname{Pr}(\mathbf{y} \mid \mathbf{z})=\left\{\begin{array}{cc}
0, & \mathbf{y}=Q[H \mathbf{z}], \\
-\infty, & \mathbf{y} \neq Q[H \mathbf{z}],
\end{array}\right.
$$

the MAP estimation in (4) can be written as the constrained optimization problem

$$
\hat{\mathbf{z}}=\arg \min _{\mathbf{z} \in \mathcal{Z}}\{-\log \operatorname{Pr}(\mathbf{z})\}
$$

where $\mathcal{Z}$ is the set of images which compress to $\mathbf{y}$ (i.e. $\mathcal{Z}=\{\mathbf{z}: \mathbf{y}=Q[H z]\}$ ).

\section{B. Image model}

For a model of a "good" image (i.e. $\operatorname{Pr}(\mathbf{z})$ ) a nonGaussian Markov random field (MRF) model is used [9; 10]. This model has been shown to successfully model both the smooth regions and discontinuities present in images.
A Gibbs distribution is used to explicitly write the distribution of MRF's. A Gibbs distribution is any distribution which can be expressed in the form

$$
\operatorname{Pr}(\mathbf{x})=\frac{1}{Z} \exp \left\{-\sum_{c \in \mathcal{C}} V_{c}(\mathbf{x})\right\}
$$

where $Z$ is a normalizing constant, $V_{c}(\cdot)$ is any function of a local group of points $c$ and $\mathcal{C}$ is the set of all such local groups. Note that the Gaussian model is a special case of the MRF. To understand how to include discontinuities into the statistical model, it is important to first understand what the model represents and how to define the model for a particular application. For a particular source signal $\mathbf{z}_{1}$, the value of the probability measure $\operatorname{Pr}\left(\mathbf{z}_{1}\right)$ is related to how closely $\mathbf{z}_{1}$ matches our prior information about the source. So a $z_{1}$ which closely matches our prior information should have a higher probability than one that does not. For this to be true, the function $V_{c}(\cdot)$ should provide a measure of the consistency of a particular $z$, where a $\boldsymbol{z}$ more consistent with the prior information will have smaller values of $V_{c}(\cdot)$. The situation which is important in this work occurs when the prior information is mostly true but a limited amount of inconsistency is allowable (e.g. a piecewise smooth surface; that is, a surface which is mostly smooth but a few discontinuities are allowable).

In this research a special form of the MRF is used which has this very desirable property. This model is characterized by a special form of the Gibbs distribution

$$
\operatorname{Pr}(\mathbf{x})=\frac{1}{Z} \exp \left\{-\frac{1}{\lambda} \sum_{c \in \mathcal{C}} \rho_{T}\left(\mathbf{d}_{c}^{t} \mathbf{x}\right)\right\}
$$

where $\lambda$ is a scalar constant that is greater than zero, $\mathbf{d}_{c}$ is a collection of linear operators and the function $\rho_{T}(\cdot)$ is given by

$$
\rho_{T}(u)= \begin{cases}u^{2}, & |u| \leq T \\ T^{2}+2 T(|u|-T), & |u|>T\end{cases}
$$

see Figure 1. Since $\rho_{T}(\cdot)$ is convex, this particular form of the MRF results in a convex optimization problem when used in the MAP estimation formulation (4). Therefore, such MAP estimates will be unique, stable, and can be computed efficiently. The function $\rho_{T}(\cdot)$ is known as the Huber minimax function [13] and for that reason this statistical model is called the Huber-Markov random field model (HMRF).

For this distribution, the linear operators, $\mathbf{d}_{c}$, provide the mechanism for incorporating what is considered consistent most of the time while the function $\rho_{T}(\cdot)$ is the mechanism for allowing some inconsistency. The parameter $T$ controls the amount of inconsistency allowable. The function $\rho_{T}(\cdot)$ allows some inconsistency by reducing the importance of the consistency measure when the value of the consistency measure exceeds some threshold, $T$.

For the measure of consistency, the fact that the difference between a pixel and its local neighbors should be small is used; that is, there should be little local variation 


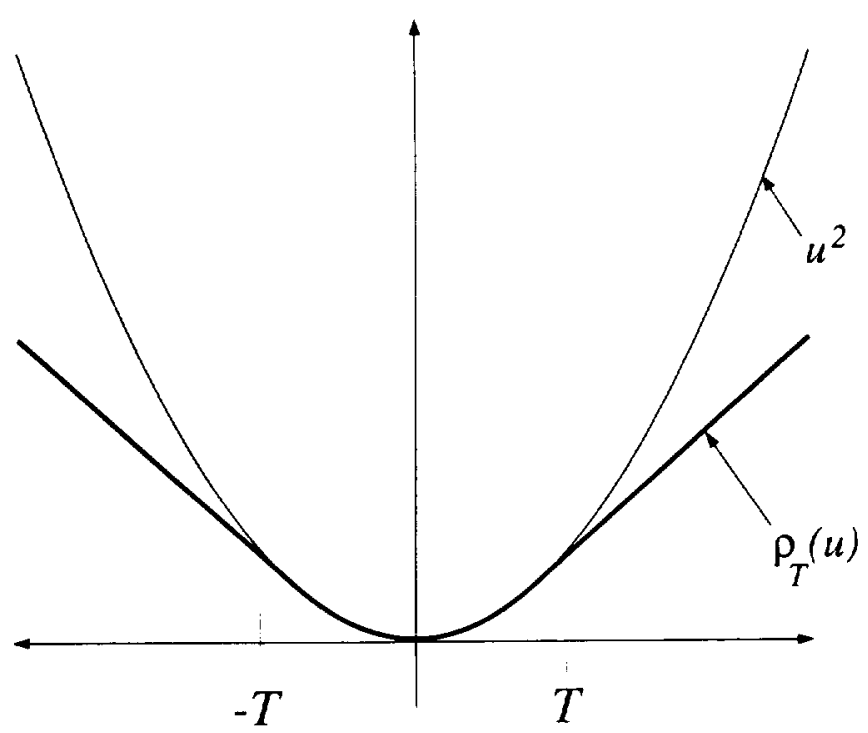

Fig. 1. Huber minimax function $\rho_{T}(\cdot)$ superimposed on quadratic func tion

in the image. For this assurnption, an appropriate set of consistency measures is

$$
\left\{\mathbf{d}_{c}^{t} \mathbf{z}\right\}_{c \in \mathcal{C}}=\left\{\mathbf{z}_{m, n}-\mathbf{z}_{k, l}\right\}_{k, l \in \mathcal{V}_{m, n, 1} \leq m, n \leq N}
$$

where $\mathcal{N}_{m, n}$ consists of the eight nearest neighbors of the pixel located at $(m, n)$ and $N$ is the dimension of the image. Across discontinuities this measure is large, but the relative importance of the measure at such a point is reduced because of the the use of the Huber function.

The MAP estimate can now be written as

$$
\begin{aligned}
\hat{\mathbf{z}} & =\arg \min _{\mathbf{z} \in \mathcal{Z}} \sum_{c \in \mathcal{C}} V_{c}(\mathbf{z}) \\
& =\arg \min _{\mathbf{z} \in \mathcal{Z}} \sum_{1 \leq m, n \leq N} \sum_{k, l \in \mathcal{N}_{m, n}} \rho_{T}\left(\mathbf{z}_{m, n}-\mathbf{z}_{k, l}\right) .
\end{aligned}
$$

As a result of the choice of image model $[9 ; 10]$, this results in a convex (but not quadratic) constrained optimization which can be solved using iterative techniques.

\section{RECONSTRUCTION ALGORITHM}

An iterative approach is used to find $\hat{\mathbf{z}}$ in the constrained minimization of (15). An initial estimate $z^{(0)}$ is improved by successive iterations until the difference between $\mathbf{z}^{(k)}$ and $\mathbf{z}^{(k+1)}$ is below a given threshold $\epsilon$. The rate of convergence of the iteration is affected by the choice of the initial estimate. A better initial estimate will result in faster convergence. The initial estimate used here is formed by the standard decompression

$$
\mathbf{z}^{(0)}=H^{-1} Q^{-1}[\mathbf{y}]
$$

Given the estimate at the $k$-th iteration, $\mathrm{z}^{(k)}$, the gradient descent method is used to find the estimate at the next iteration, $\mathbf{z}^{(k+1)}$. The gradient of $\sum \rho_{T}\left(\mathbf{d}_{c}^{t} \mathbf{z}\right)$ is used

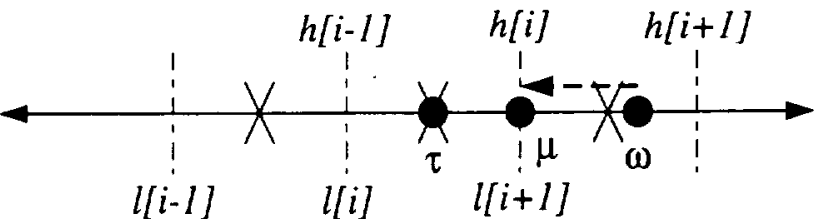

Fig. 2. Illustration of projection operator for scalar quantization.

to find the steepest direction $\mathbf{g}^{(k)}$ towards the minimum

$$
\mathbf{g}^{(k)}=\nabla\left(\sum_{c \in \mathcal{C}} \rho_{T}\left(\mathbf{d}_{c}^{t} \mathbf{z}^{(k)}\right)\right)=\sum_{c \in \mathcal{C}} \rho_{T}^{\prime}\left(\mathbf{d}_{c}^{t} \mathbf{z}^{(k)}\right) \mathbf{d}_{c}^{t},
$$

where $\rho_{T}^{\prime}(u)$ is the first derivative of the Huber function. The size of the step $\alpha^{(k)}$ is chosen as

$$
\alpha^{(k)}=\frac{\mathbf{g}^{(k) t} \mathbf{g}^{(k)}}{\mathbf{g}^{(k) t}\left(\sum_{c \in \mathcal{C}} \rho_{T}^{\prime \prime}\left(\mathbf{d}_{c}^{t} \mathbf{z}^{(k)}\right) \mathbf{d}_{c} \mathbf{d}_{c}^{t}\right) \mathbf{g}^{(k)}} .
$$

This choice of step size is based on selecting the optimal step size for a quadratic approximation to the nonquadratic function in (15). Since this is an approximation, the value of the objective function may increase if the step size is too large. To avoid this potential problem, the value of $\alpha^{(k)}$ is divided by 2 until the step size is small enough that the value of the objective function is decreased. Since the updated estimate $\mathbf{w}^{(k+1)}$,

$$
\mathbf{w}^{(k+1)}=\mathbf{z}^{(k)}+\alpha^{(k)} \mathbf{g}^{(k)},
$$

may fall outside the constraint space $\mathcal{Z}, \mathbf{w}^{(k+1)}$ is projected onto $\mathcal{Z}$ to give the image estimate at the $(k+1)$-th iteration

$$
\mathbf{z}^{(k+1)}=\mathcal{P}_{2}\left(\mathbf{w}^{(k+1)}\right) \text {. }
$$

In projecting the image $\mathbf{w}^{(k+1)}$ onto the constraint space $\mathcal{Z}$, we are finding the point $\mathbf{z}^{(k+1)} \in \mathcal{Z}$ for which $\| \mathbf{z}^{(k+1)}-$ $\mathbf{w}^{(k+1)} \|$ is a minimum. If $\mathbf{w}^{(k+1)} \in \mathcal{Z}$, then $\mathbf{z}^{(k+1)}=$ $\mathbf{w}^{(k+1)}$ and $\left\|\mathbf{z}^{(k+1)}-\mathbf{w}^{(k+1)}\right\|=0$. Since $H$ is unitary,

$$
\left\|H \mathbf{z}^{(k+1)}-H \mathbf{w}^{(k+1)}\right\|=\left\|\mathbf{z}^{(k+1)}-\mathbf{w}^{(k+1)}\right\|
$$

and the projection can be carried out in the transform domain.

The form of $\mathcal{P}_{\mathcal{Z}}$ is dependent on the quantizer $Q$. The projection operators $\mathcal{P}_{\mathcal{Z}}$ for scalar and vector quantization are described in the following subsections.

\section{A. Scalar quantization}

The scaler quantizer is a partition of the real number line $\mathcal{R}$ and is defined by its breakpoints. The breakpoints are determined by the amount of compression desired. The boundaries or breakpoints of cell $i$ are $l[i]$ and $h[i]$, see Figure 2. If a coefficient $\omega$ falls in cell $i$,

$$
l[i] \leq \omega<h[i]
$$

then index $i$ is transmitted. The compressed representation $y$ is the set of indices for all the transform coefficients. 
Projection to the constraint space is rather simple for scalar quantization. To find the transform coefficients $\mu$ in $H \mathbf{z}^{(k+1)}$, find the corresponding coefficient $\omega$ in $H \mathbf{w}^{(k+1)}$. From the compressed representation $\mathbf{y}$, find in which cell $\mu$ should fall. Assume $\mu$ should fall in cell $i$. The standard decompression method would use $\mu=\tau$ where $\tau$ is the centroid of the cell. The coefficient $\mu$ which falls in cell $i$ can be determined by

$$
\mu=\left\{\begin{array}{cl}
l[i], & \omega<l[i], \\
\omega, & l[i] \leq \omega<h[i] \\
h[i], & h[i] \leq \omega .
\end{array}\right.
$$

This will minimize $\left\|H \mathbf{z}^{(k+1)}-H \mathbf{w}^{(k+1)}\right\|$ while assuring that $\mathbf{y}=Q\left[H \mathbf{z}^{(k+1)}\right]$. The image estimate $\mathbf{z}^{(k+1)}$ is obtained by performing the inverse transformation on $H \mathbf{z}^{(k+1)}$.

\section{B. Vector quantization}

The vector quantizer is a partition of $\mathcal{R}^{n}$ and is defined

- by its codebook vectors $\tau[i]$. The size of the codebook is determined by the desired bit rate for that subband.

A coefficient vector wis considered in cell $i$ if it is closer to codevector $\boldsymbol{\tau}[i]$ than to any other codevector

$$
\|\boldsymbol{\omega}-\tau[i]\|<\|\boldsymbol{\omega}-\tau[j]\|, \quad j \neq i .
$$

- The distance metric used here is the squared Euclidean distance. Although the illustrations in Figure 3 represent a 2-D quantizer, the method described below for finding $\boldsymbol{\mu}$ directly generalizes to higher dimensional spaces.

Projection to the constraint space is more complicated for VQ than for the scalar case. The standard decompression method would use $\boldsymbol{\mu}=\boldsymbol{\tau}[i]$ for the reconstruction point. To project the vector $\boldsymbol{\omega}$ to cell $i$, it is necessary to find the point in cell $i$ which is closest to $\omega$. The easy case occurs when $\boldsymbol{\omega}$ itself is in cell $i$ since $\boldsymbol{\mu}=\boldsymbol{\omega}$. In the more difficult case where $\omega$ is closer to some other codevector, the projection point $\boldsymbol{\mu}$ will be on the boundary of cell $i$. The first step in projecting $\boldsymbol{\omega}$ to $\boldsymbol{\mu}$ is to move from $\boldsymbol{\omega}$ to the boundary of cell $i$. The next step is to move along the boundary to $\boldsymbol{\mu}$.

The points $\boldsymbol{\xi}$ on the boundary of cell $i$ all satisfy

$$
\|\xi-\tau[i]\|=\|\xi-\tau[j]\|
$$

for some $j \neq i$. For a particular value of $j,(25)$ defines a hyperplane of points equally distant from the $i$-th and $j$-th codevectors. Since $\omega$ is outside cell $i$, the line segment from $\boldsymbol{\omega}$ to $\boldsymbol{\tau}[i]$ described by

$$
\xi=t \omega+(1-t) \tau[i], \quad 0 \leq t \leq 1
$$

will intersect the boundary of cell $i$. For each codevector $\tau[j], \quad j \neq i$, it is easy to determine if the intersection of the hyperplane (25) with the line segment (26) exists. The value of $t$ in (26) which defines the point of intersection is given by

$$
t=\frac{(\tau[i]-\tau[j]) \cdot(\tau[i]-\tau[j])}{2(\tau[i]-\omega) \cdot(\tau[i]-\tau[j])}
$$
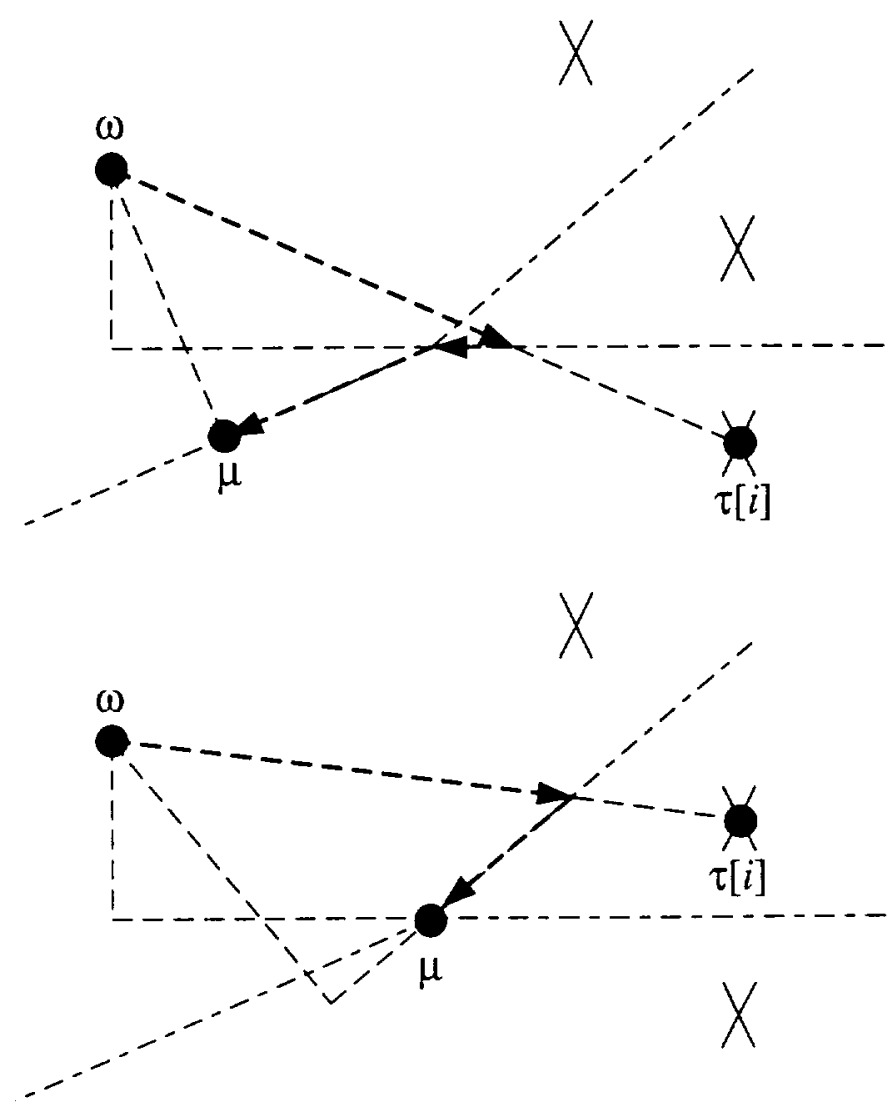

Fig. 3. Illustration of projection operator for vector quantization

where $t \in[0,1]$ if the intersection exists. Among all $j \neq i$, the smallest positive value of $t$ will define the point $\xi_{1}$ on the cell boundary. $\xi_{1}$ will be equally distant from $\tau[i]$ and $\tau[k]$.

The next step involves traveling along the boundary of the cell to $\boldsymbol{\mu}$. The idea is to move towards $\boldsymbol{\omega}$ but not leave the cell boundary. Let $\boldsymbol{\xi}_{2}$ be the perpendicular projection of $\boldsymbol{\omega}$ onto the $k$-th hyperplane $(\|\boldsymbol{\xi}-\tau[i]\|=\|\xi-\tau[k]\|)$. $\boldsymbol{\xi}_{2}$ is the point in this hyperplane which is closest to $\boldsymbol{\omega}$ and can also be found as the intersection of a line

$$
\xi=\omega+t(\tau[i]-\tau[k])
$$

with a hyperplane. Both $\xi_{1}$ and $\xi_{2}$ will be in this hyperplane. Next, move along the line segment from $\xi_{1}$ towards $\boldsymbol{\xi}_{2}$, stopping at the first intersecting hyperplane. The intersection point becomes the new $\boldsymbol{\xi}_{1}$ and this hyperplane becomes the new $k$-th hyperplane. $\xi_{2}$ now becomes the projection of $\boldsymbol{\omega}$ onto this new hyperplane and the process is repeated until one of the stopping conditions is met. This process is stopped if

- $\xi_{1}$ travels all the way to $\xi_{2}$ without being intercepted by another hyperplane

- or if $\xi_{1}$ would leave the cell boundary by moving towards $\xi_{2}$

- or if $\boldsymbol{\xi}_{1}$ does not make progress towards $\boldsymbol{\omega}$.

When this process is completed, $\boldsymbol{\mu}=\boldsymbol{\xi}_{1}$ 


\section{EXPERIMENTAL RESULTS}

In this section, the results of using the proposed postprocessing method are shown for images compressed using scalar quantization of block DCT and using vector quantization of subband wavelet transform.

\section{A. Scalar quantization}

The standard "Lenna" image shown in Figures 4(a) and 5 (a) was compressed to 0.264 bpp by the JPEG algorithm $[11 ; 1]$. The result of standard decompression of this image is shown in Figure 4(b) and enlarged to show detail in Figure 5(b). At this compression ratio, the coding artifacts are very noticeable. Most noticeable are the blocking effects of the coding algorithm, but aliasing artifacts along large contrast edges are also noticeable. The result of postprocessing this image is shown in Figure 4(c) and enlarged to show detail in Figure $5(\mathrm{c})$. The number of iterations needed to sufficiently reduce the artifacts is dependent on the severity of the degradation. More iterations are required for con-

- vergence with more severe degradation. Notice that the blocking effects have been removed in the postprocessed image. This can be most easily seen in the shoulder and background regions. Notice that while the discontinuities due to the blocking effects have been smoothed, the sharp discontinuities in the original image, such as along the hat brim, have been preserved.

For comparison, the method for reducing blocking artifacts proposed in the JPEG standard [1] is shown in Figures $4(\mathrm{~d})$ and $5(\mathrm{~d})$. For a given block, the five lowest - frequency $\mathrm{AC}$ coefficients are predicted from the DC coefficients of the given block and the 8 nearest blocks. The image is modeled as a 2-D second order polynomial over these 9 blocks. This polynomial is defined by requiring that the mean of the polynomial over each block must match the DC value for that block. To reduce the blocking, the estimated $\mathrm{AC}$ coefficients corresponding to a DCT of this polynomial are used if they do not contradict the transmitted information. See [1] for details. Note that while the blocking artifacts are reduced by this approach, they are still visible. The overall improvement in the reconstructed image is much less significant than the improvement seen in Figures 4(c) and 5(c).

While the blocking artifact dominated the standard reconstruction of the "Lenna" image, ringing artifacts are dominant in document images such as text or graphics [14], see Figure 6. While JPEG may not be the most appropriate compression method for document images, it can be seen from the example shown in Figure 6 that ringing artifacts are suppressed by the postprocessing algorithm. The postprocessing algorithm removes ringing artifacts as well as blocking artifacts.

\section{B. Vector quantization}

The "Lenna" image was decomposed in the manner of Mallat [15] by a three level separable subband structure using 2-D separable filters based on 1-D Daubechies' 12-th order wavelet filters [16]. Each of the subbands is vector quantized quantized independently with the transform co- efficients grouped into 4 by 4 blocks (or 8 by 8 ) which are treated as 16 (or 64) dimensional vectors. The bit rate for each subband is determined by the method given in [12] based on the desired overall bit rate and human visual system considerations. The bit rate is higher for lower frequency subbands. The highest frequency subbands are allocated zero bpp and are dropped altogether. For each of the remaining subbands, a locally optimal VQ codebook is designed for that subband signal. See [17] for general information on VQ and [12] for details on the VQ approach which is used here. The point here is to demonstrate the postprocessing technique is sufficiently flexible to be applied to any transform coding method.

Figure 7 shows the results for moderate compression $(0.444 \mathrm{bpp})$ and postprocessing for 9 iterations. The results of postprocessing for 20 iterations are shown in Figure 8 for the case of heavy compression ( $0.155 \mathrm{bpp})$. The greatest improvement comes in the first few iterations. Further iterations of postprocessing continue to improve the image, but the amount of improvement in each iteration decreases as the image gets better.

The primary artifacts of this coding technique are aliasing at edges and loss of high frequency information. As expected, these artifacts are more severe in the case of heavy compression. Most aliasing artifacts disappear after only a few iterations in the case of moderate compression. Although the aliasing artifacts are more persistent for heavy compression, the first few iterations still reduce the aliasing a great deal. Even for heavy compression, the aliasing artifacts are eventually no longer perceptible.

Information contained in high frequency subbands, such as the details in the hat of the original image, is completely lost when those subbands are dropped. The postprocessing cannot reinvent this information which is completely absent from the compressed representation. However, for structures such as edges which have information content spread across low frequencies as well as high frequencies, the postprocessor is able to restore some high frequency information based on the low frequency components in the compressed representation. This allows crisper edges which contain high frequencies to appear in the restored image. See for example the hat brim and along the edge of the shoulder. As in the scalar quantization case, the structures supported by the compressed representation and by the image model are restored while artifacts of the transform coding process are suppressed.

\section{CONCLUSION}

The problem of image decompression has been cast as an ill-posed inverse problem, and a stochastic regularization technique has been used to form a well-posed reconstruction algorithm. A statistical model for the image was produced which incorporated the convex Huber minimax function. The use of the Huber minimax function $\rho_{T}(\cdot)$ helps to maintain the discontinuities from the original image which produces high resolution edge boundaries. Since $\rho_{T}(\cdot)$ is convex, the resulting multidimensional minimization problem is a constrained convex optimization problem. 
(a)
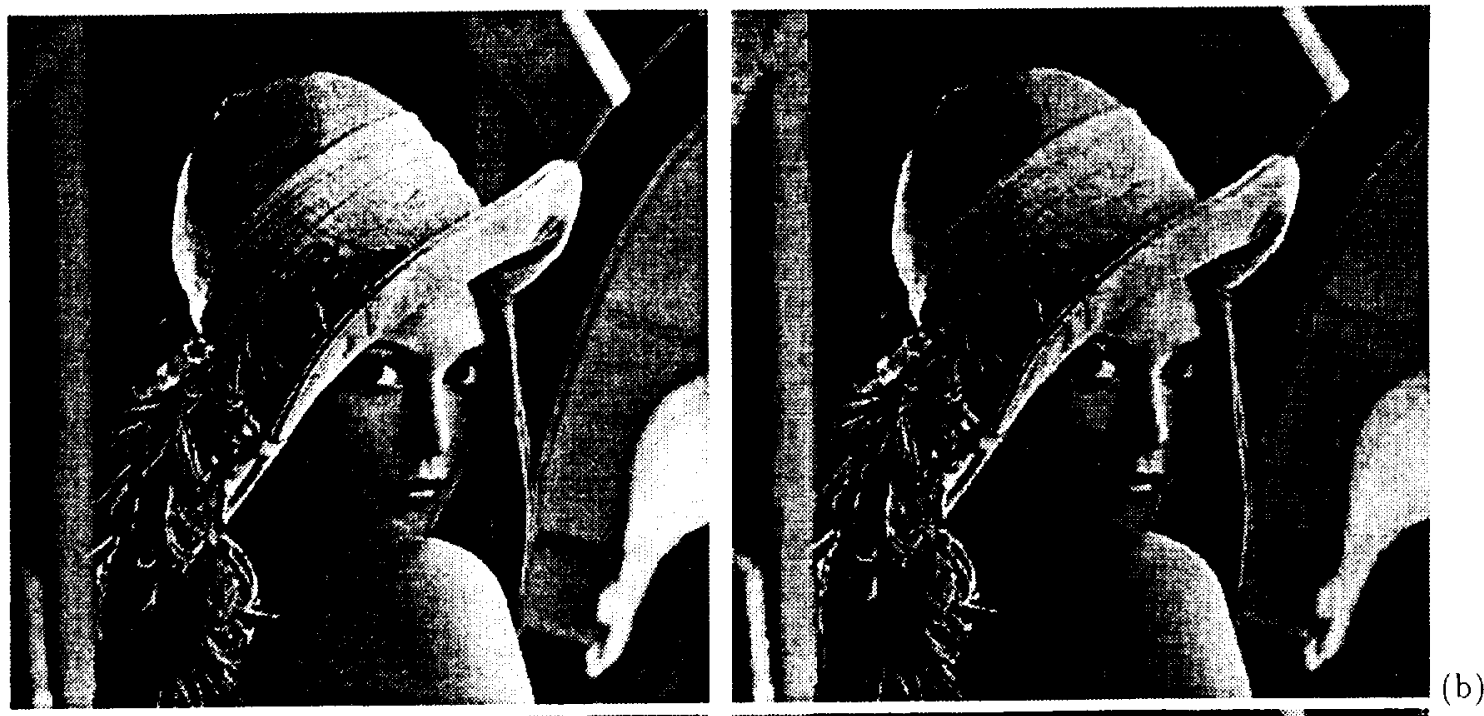

(c)
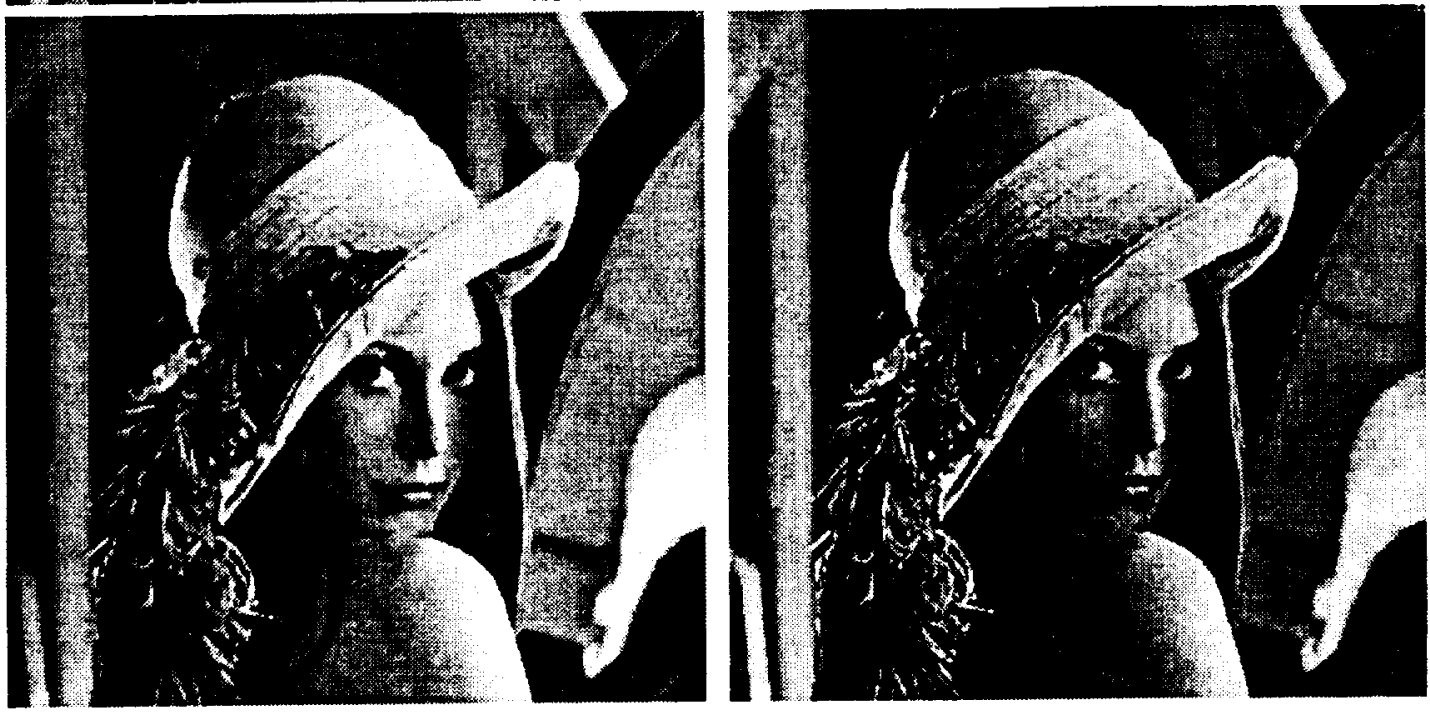

(b)

(d)

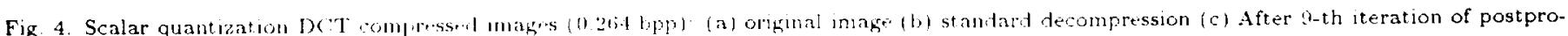

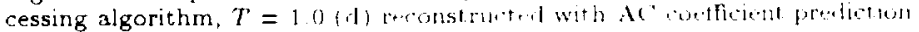

Efficient computational algorithus can be used in the minimization. The proposed image decompression algorithm produces reconstructed innges which grratly reduced the noticeable artifacts which exist using standard decompression techniques.

\section{REFEREN('ES}

[1] W. B. Pennebaker and J. L. Mitchell, JPlGG: Still Lmage Data Compression Standurd. New York: Van Nostrand Reinhold, 1993.

[2] R. L. Stevenson. "IReduction of coding artifact.s in transform image coding," in Proccodings of the 199:3 International Conferruces on . Loustics. Sifuech and Signal Processing, (Minneapolis, MN). Pr. V.101 10.4. Apr. 1993.

[3] T. P. O'Rourke and R. L. Stevenson, "Inproverl inage decompression for recluwed transform colling artifacts," in Pror. SPIE lmaye and Video Processin!ll II.

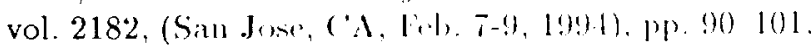
1994.
[4] H. C. Reeve and J. S. Lim, "Reduction of blocking effects in image coding," Optical Engineering, vol. 23, pp. 34-33. Jan./Feb. 1984.

[5] K. N. Ngan, D. W. Lin, and J. T. Liou, "Enhancement of innage quality for low bit rate video coding," IEEE Trans. on Circuits and Systems, vol. 38, pp. 12211225. Oct. 1991.

[6] R. Rosinholtz and $A$. Zakhor, "Iterative procedures for reduction of blocking effects in transform image coding," IEEE Trans. on Circuits and Systems for Video Technology, vol. 2, pp. 91-95, Mar. 1992.

[7] B. Ramamurthi and A. Gersho, "Nonlinear spacevariant postprocessing of block coded images," IEEE Trums. on Acoustics. Speech, and Signal Processing, vol. ASSP-3-4, pp. 1258-1268, Oct. 1986.

[8] K. Sautr, "Enhancement of low bit-rate coded images using olge detection and estimation," Computer Vision (iriphics and Image Processing: Graphical Modfls and Image Processing. vol. 53, pp. 52-62, Jan. 1991 

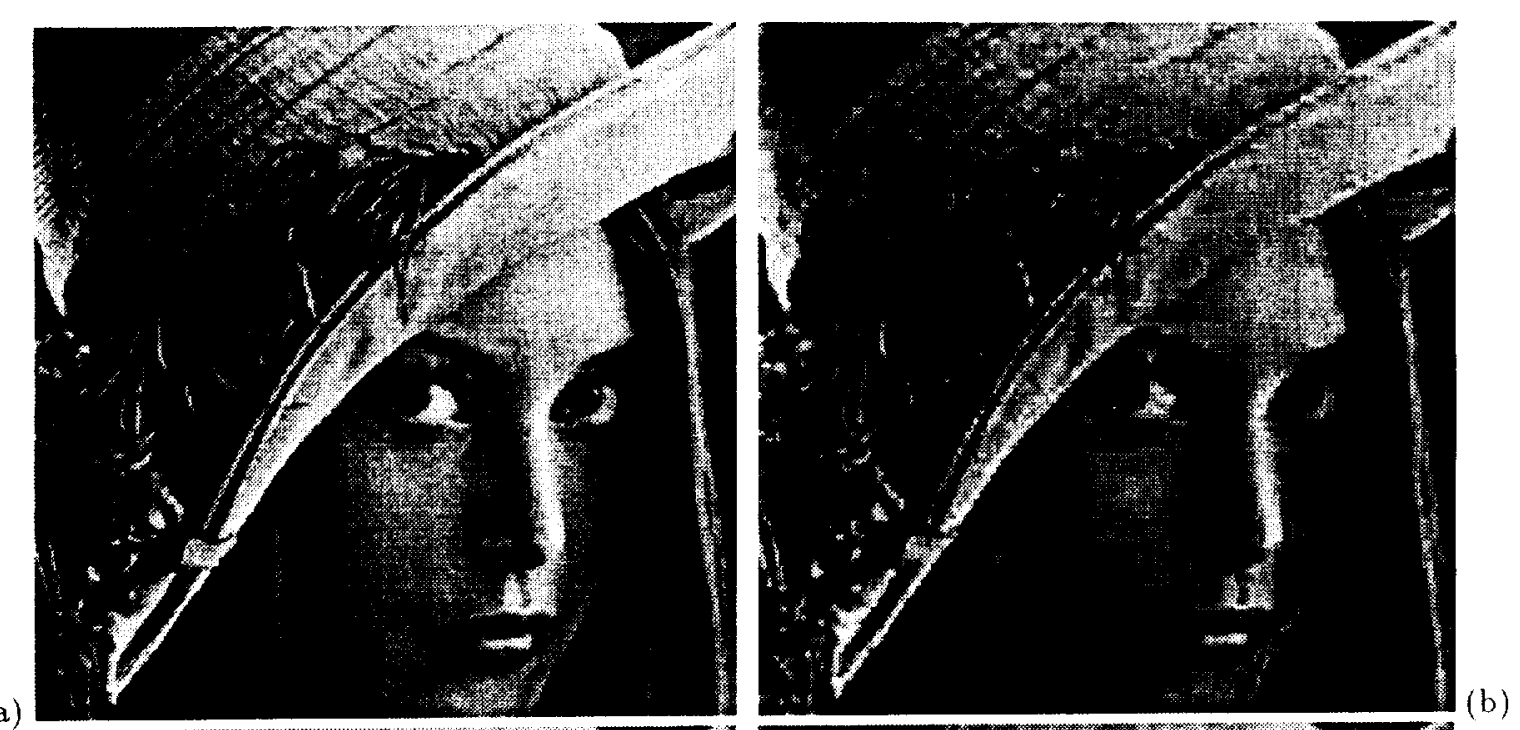

(c)
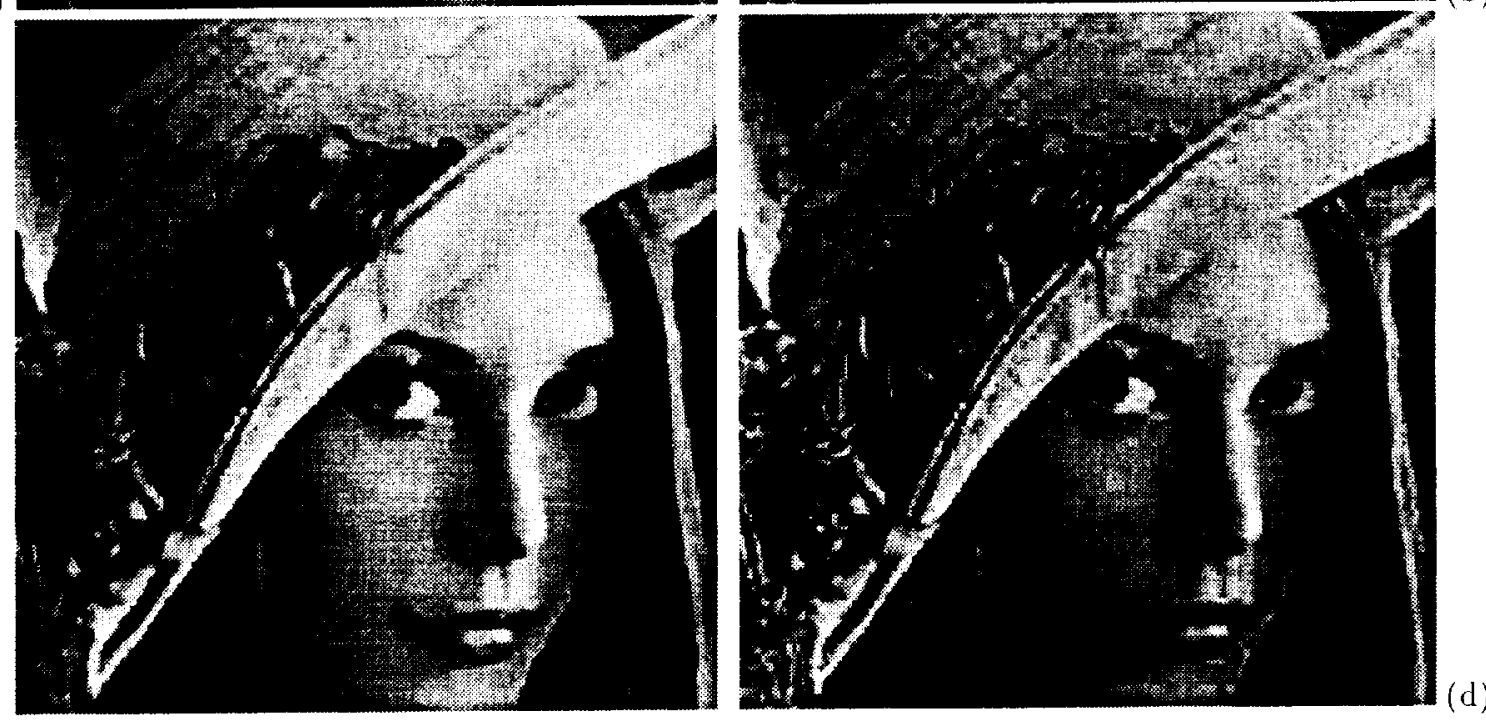

Fig. 5. Scalar quantization DCT ampressed images (0) 2f.4 tpp (enlarged view) (a) original image (b) standard decompression (c) After 9-th iteration of postprocessing algorithm, $T=1.0$ (1) reconstructed with AC coefficient prediction

[9] R. R. Schultz and R. L. Stevenson, "Improved definition image expansion," in Proceedings of the 1992 International Conference on Acoustics, sipech and Signal Processing, (San Francisco, CA), pp. III:173-176, Mar. 1992.

[10] R. L. Stevenson and S. M. Schweizer, "A nonlinear filtering structure for inlage smoothing in mixed noise environments," Journal of Mathematical Imaging and Vision, vol. 2, pp. 137-154, Nov. 1992.

- [11] G. K. Wallace, "The JPEG picture compression standard," IEEE Trans. on Consumer Electronics, vol. 38, pp. 18-34, Feb. 1992.

- $\quad$ 12] T. P. O'Rourke and R. L. Stevenson, "IIuman visual system based subband image compression." in Proc. 31st Annual Allerton Conference on Communucation. Control, and Computing. (Monticello, IL. Sept. 29Oct. 1, 1993), pp. 452 461,19933 .

[13] P. J. Huber, Robust Statestics. New York: Jolun Wiley \& Sons, 1981 .

[14] Z. Fan and R. Esclibach. "JPECi decompression with reduced artifacts," in Proc. of ISE T/SPIE Symposium on Electronic Imaging: Image and Video Compression, (San Jose, CA, Feb. 6-10, 1994), 1994.

[15] S. (. Mallat, "A theory for multiresolution signal decomposition: The wavelet representation," IEEE Trans. on Pattern Analysis and Machine Intelligence, vol. 11, pp. 674-693, July 1989.

[16] I. Daubechies, "Orthonormal bases of compactly supported wavelets," Communications on Pure and Applied Mathematics, vol. XLI, pp. 909-996, 1988.

[17] A. Gersho and R. M. Gray, Vector Quantization and Signal Compression. Boston: Kluwer Academic Publishers, 1992. 
- he ucpal hilenl

The Laboratory 010101

Workstations, e

monitors and $\mathrm{p}, \mathrm{S}$,

i ile ucpal minil

The Laboratory 01.019

Workstations, e

cameras, frame $\mathrm{A}, \mathrm{Pa}$.

i He uepal uneil

The Laboratory 01101

Workstations, e

cameras, frame

monitors and $\mathrm{p} 1 \mathrm{~s}$

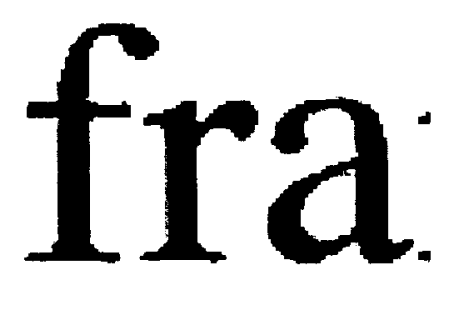

Fig. 6. Scalar quantization DCT compressed images (0.264 tpp): (a) original image (b) enlarged (c) standard decompression with ringing artifacts (d) enlarged (e) After postprocessing algorithm, $T=1.0$ (f) enlarged. Note for revewers: halftoning artifacts distort the letter edges. This distortion is not present in the original images and will not appear in the glossy images in the final manuscript. 
(a)

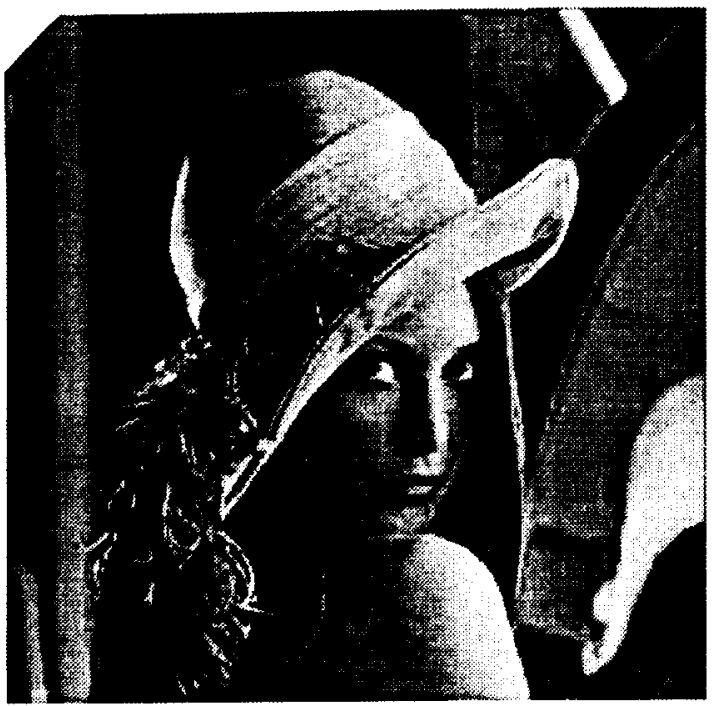

(c)

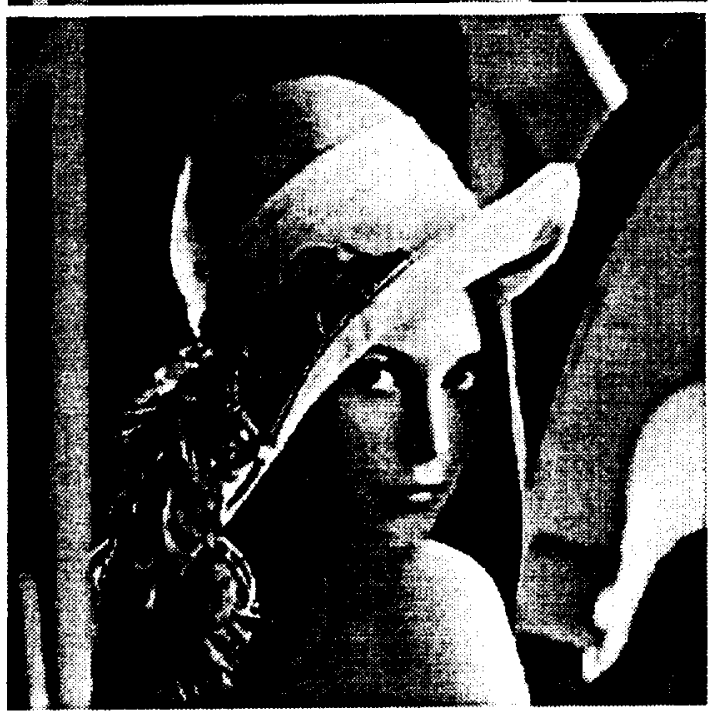

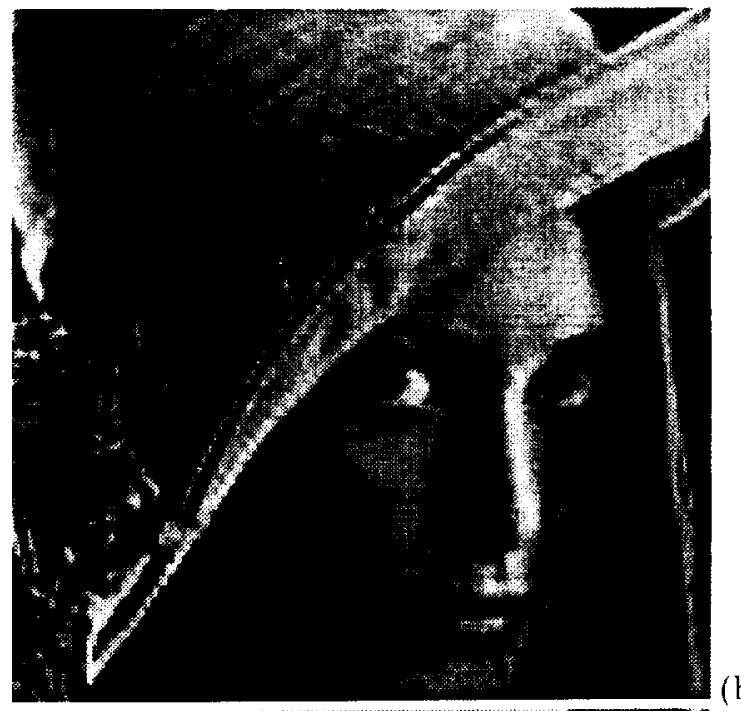

(b)

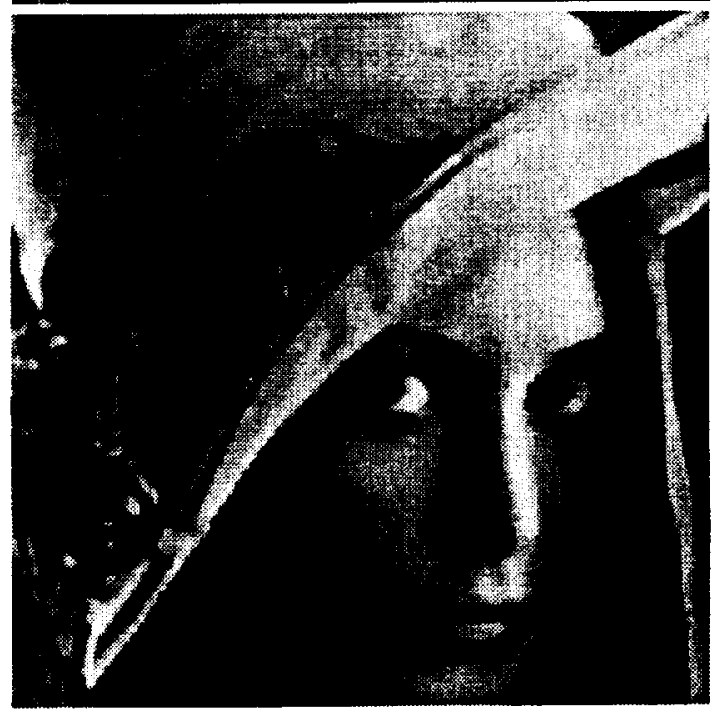

(d)

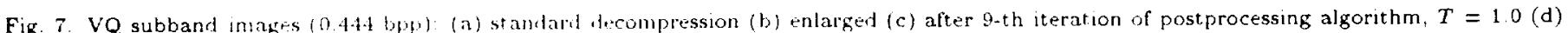
enlarged.

Thomats P. O Ronrke repeivet the B.S.F.E. degree (with honors) from the University of Illims at I/drana-( Thampaign in 1900 and the M.S.E.E. from the Thiversity of Notre Dame in 1993. White at the University of [llinois he participatediut the esenerative eduation program witl IBN Corp. in Lexinglon, Kentucky He is currenty putsuing the Ph.D. in Eler: trical Enginering at the tenisersity of Notre Dame. Mr. o'Ronute is a meminer of the IFEF, the NIA. Lita Lappa Nu, Tau Beta $\mathrm{Pi}$, and Phi Kappa Phi. His researh interests include intage compression, multidimensional signal proxessing. image communication systems, and image procersing.
Robert $\mathbf{L}$. Stevenson was born on December 20. 1963, in Ridley Park. Pennsylvania. He received the B.E.E. degree (summa cum laude) from the University of Delaware in 1986, and the Ph.D. in Electrical Engineering from Purdue University in 1990. While at Purdue he was supported by a National Science Foundation Graduate Fellowship and a graduate fellowship from the DuPont Corporation. He joined the faculty of the Department of Electrical Engineering at the University of Notre Dame in 1990, where he is currently an Assistant Professor. Dr. Stevenson is a member of the IEEE, Eta Kappa Nu, Tau Beta Pi, and Phi Kappa Phi. His research interests include multidimensional signal processing, image processing, and computer vision. 


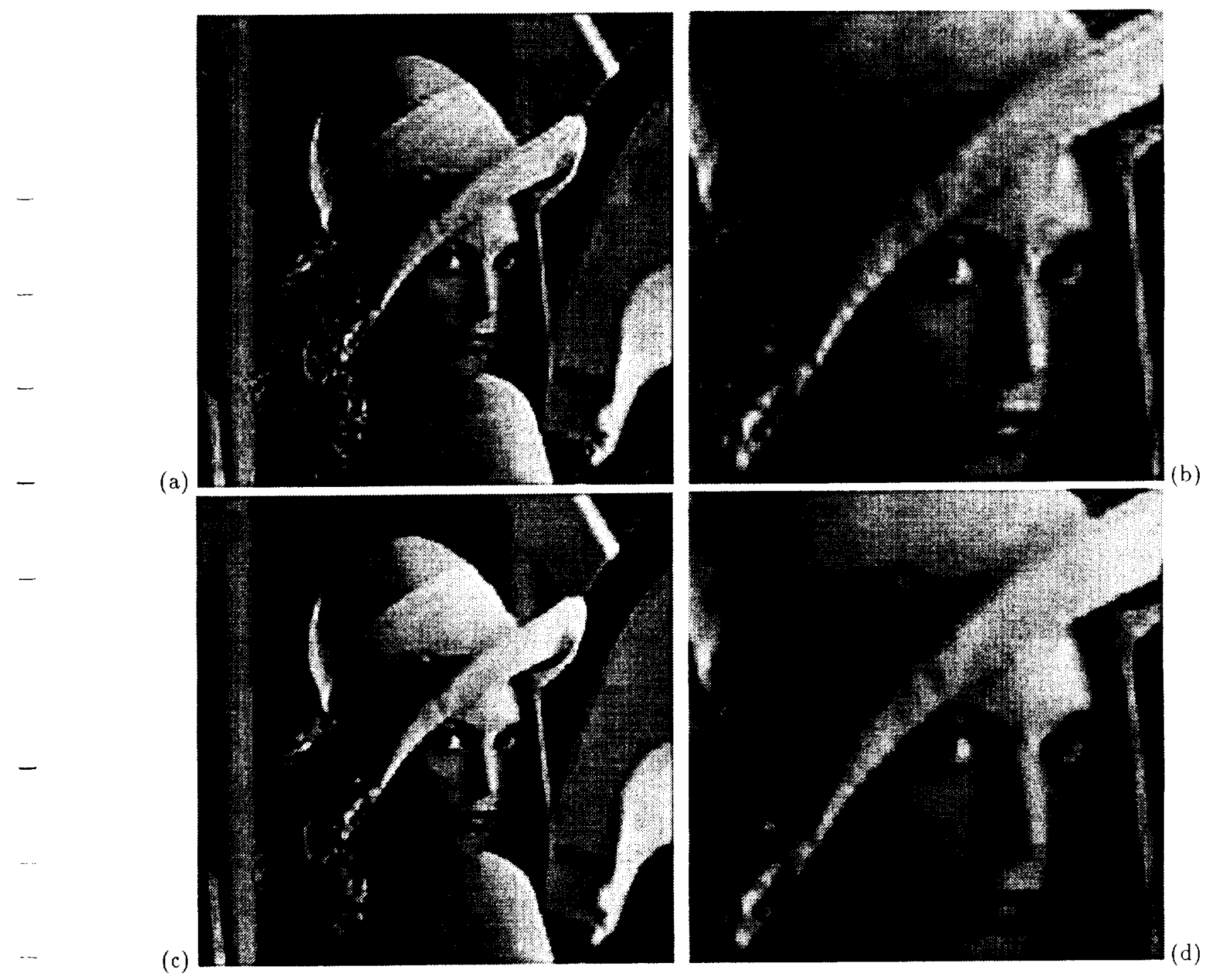

Fig. 8. VQ subband images ( $0.155 \mathrm{~h}, \mathrm{pp})$ : (a) standard decompression (b) enlarged (c) after 20-th iteration of postprocessing algorithm, $T=1.0$ (d) enlarged. 